\title{
The mineralization effect of wheat straw on soil properties described by MFPC analysis and other methods
}

\author{
Monika Jakubus ${ }^{1}$, Mirosław Krzyśko ${ }^{2,3}$, \\ Waldemar Wołyński ${ }^{3}$, Małgorzata Graczyk ${ }^{4}$
}

\footnotetext{
${ }^{1}$ Department of Soil Science and Land Protection, Poznań University of Life Sciences Szydłowska 50, 60-656 Poznań, Poland, e-mail: monja@up.poznan.pl

${ }^{2}$ Faculty of Management, President Stanisław Wojciechowski Higher Vocational State

School, Nowy Świat 4, 62-800 Kalisz, Poland, e-mail: mkrzysko@amu.edu.pl

${ }^{3}$ Faculty of Mathematics and Computer Science, Adam Mickiewicz University, Umultowska 87, 61-614 Poznań, Poland, e-mail: wolynski@amu.edu.pl

${ }^{4}$ Department of Mathematical and Statistical Methods, Poznań University of Life

Sciences, Wojska Polskiego 28, 60-637 Poznań, Poland, e-mail: magra@up.poznan.pl
}

\section{SUMMARY}

Recycling of crop residues is essential to sustain soil fertility and crop production. Despite the positive effect of straw incorporation, the slow decomposition of that organic substance is a serious issue. The aim of the study was to assess the influence of winter wheat straws with different degrees of stem solidness on the rate of decomposition and soil properties. An incubation experiment lasting 425 days was carried out in controlled conditions. To perform analyses, soil samples were collected after 7, 14, 21, $28,35,49,63,77,91,119,147,175,203,231,259,313,341,369,397$ and 425 days of incubation. The addition of two types of winter wheat straw with different degree of stem solidness into the sandy soil differentiated the experimental treatments. The results demonstrate that straw mineralization was a relatively slow process and did not depend on the degree of filling of the stem by pith. Multivariate functional principal component analysis (MFPC) gave proof of significant variation between the control soil and the soil incubated with the straws. The first functional principal component describes $48.53 \%$ and the second $18.55 \%$, of the variability of soil properties. Organic carbon, mineral nitrogen and sum of bases impact on the first functional principal component, whereas, magnesium, sum of bases and total nitrogen impact on the second functional principal component.

Key words: incubation process, multivariate functional principal component analysis, soil properties, winter wheat straws with different degree of stem solidness 


\section{Introduction}

The decline of soil organic matter, as a consequence of intense soil cultivation practices, has been identified as one of the most important threats to soil quality. To reverse these impacts, the application of organic substances has been employed. Organic substances such as straw may increase the input of carbon and nutrients. Besides, returning crop straw into fields is a common practice to resolve an oversupply of straw and improve soil organic matter. The application of such organic soil amendments is in line with the Thematic Strategy for Soil Protection (2006), where the decline of organic matter was underlined. Microbial decomposition of added crop straw is affected by many factors including soil moisture content, microbial activity, straw quality and size (Chen et al. 2014). All Polish varieties of winter wheat have hollow stems, and are susceptible to wheat stem sawfly (Cephus pygmaeus L.). Considering the harmful effects of the pest, linked to stem damage and plant yield reduction, the bringing into cultivation of varieties with stems filled by pith is reasonable, because these varieties are more resistant to the pest. However, the degree of stem solidness is closely associated with different chemical compositions of straw and may influence its mineralization rate as well as nutrient release. In this context the degree of filling of stem by pith may play a crucial role, because it is directly related to higher or lower contents of cellulose and lignin, the most abundant components of litter, which are slowly decomposed. Moreover, crop residue decomposition is affected by the physical and chemical characteristics of both the residue and the soil where the residue is incorporated. We may suspect that filling of the stem by pith may check these processes and decrease the fertilizing effect in comparison with straw with hollow stems. The aim of this paper is to show how the mineralization processes (taking place over a long period) can be analyzed with the use of MFPC and other statistical methods.

Section 2 describes the material used in the experiment presented. In the third section we develop the theory of multivariate functional principal component analysis. Section 4 contains calculation results, and in section 5 a discussion is presented.

\section{Material and methods}

The soil used in the experiment was collected from the top layer (0-25 $\mathrm{cm}$ ) of arable land. The soil was classified as lessive soil according to IUSS 
Working Group WRB (2007). Winter wheat straw was added to the soil at doses corresponding to 0 (control soil, T0) and $6 \mathrm{Mg}$ of dry matter per hectare (T1-straw with stem filled by pith, T2-straw with hollow stem). Each combination was made in four replications for particular terms. Samples of $400 \mathrm{~g}$ dried soil were weighed in duplicate and mixed with the dose of straw. Additionally, nitrogen in the form of urea was applied to correct the C:N ratio in the soil. Each mixture was wetted to $60 \%$ field capacity and placed in $0.5 \mathrm{~L}$ plastic boxes. The samples were incubated at $25^{\circ} \mathrm{C}$ for 425 days. The particular replications of each experimental treatment were eliminated, dried and then subjected to analysis. The measurements were recorded at 20 incubation dates (terms) corresponding to the following days from the beginning of the experiment: $7,14,21,28,35,49,63,77,91,119$, $147,175,203,231,259,313,341,369,397$ and 425 . The following properties (statistical variables) were determined:

1. physiochemical: soil reaction $(\mathrm{pH})$, hydrolytic acidity $(\mathrm{Hh})$, sum of bases (SB)

2. chemical: content of organic carbon (Corg), total nitrogen (Ntot), amounts of available phosphorous, potassium, magnesium, mineral nitrogen (sum of $\mathrm{N}-\mathrm{NH}_{4}$ and $\mathrm{N}-\mathrm{NO}_{3}$ ) and available microlements (Fe, $\mathrm{Mn}, \mathrm{Zn}, \mathrm{Cu}, \mathrm{Ni})$.

The above properties were determined with the use of methods commonly applied in soil science analyses; their detailed descriptions can be found in Jakubus (2013).

\section{Multivariate functional principal component analysis}

The character of the data justifies the use of vector stochastic processes and the need for summarizing of the data into a few functions of the original components of these processes (functional principal components). The functional case of PCA (FPCA) is a more informative way of looking at the variability structure in the variance-covariance function for onedimensional functional data (Górecki and Krzyśko, 2012). In this section we present PCA for multivariate functional data (MFPCA) (Jacques and Preda 2014). Suppose that we are observing a $p$-dimensional stochastic process $\boldsymbol{X}(t)=\left(X_{1}(t), X_{2}(t), \ldots, X_{p}(t)\right)^{\prime}$, with continuous parameter $t \in I$. We will further assume that $\mathrm{E}(\boldsymbol{X}(\mathrm{t}))=\mathbf{0}$ and $\boldsymbol{X}(t) \in L_{p}^{2}(I)$, where $L_{p}^{2}(I)$ is a Hilbert space of square integrable functions on the interval $I$ equipped with the following inner product: 


$$
<\mathbf{u}(t), \mathbf{v}(t)>=\int_{I} \mathbf{u}^{\prime}(t) \mathbf{v}(t) d t .
$$

Moreover, assume that the $k$ th component of process $\boldsymbol{X}(t)$ can be represented by a finite number of orthonormal basis functions $\left\{\phi_{b}\right\}$

$$
X_{k}(t)=\sum_{b=0}^{B_{k}} c_{k b} \phi_{b}(t), t \in I, k=1,2, \ldots, p,
$$

where $c_{k b}$ are random variables such, that $\mathrm{E}\left(c_{k b}\right)=0, \operatorname{Var}\left(c_{k b}\right)<\infty$, $k=1,2, \ldots, b, b=0,1, \ldots, B_{k}$. Let

$$
\begin{aligned}
& \mathbf{c}=\left(c_{10}, \ldots, c_{1 B_{1}}, \ldots, c_{p 0}, \ldots, c_{p B_{p}}\right)^{\prime}, \\
& \boldsymbol{\Phi}(t)=\left[\begin{array}{cccc}
\boldsymbol{\phi}_{1}^{\prime}(t) & \mathbf{0} & \ldots & \mathbf{0} \\
\mathbf{0} & \boldsymbol{\phi}_{2}^{\prime}(t) & \ldots & \mathbf{0} \\
. . & \ldots & \ldots & \ldots \\
\mathbf{0} & \mathbf{0} & \ldots & \boldsymbol{\phi}_{p}^{\prime}(t)
\end{array}\right],
\end{aligned}
$$

where $\boldsymbol{\phi}_{k}(t)=\left(\phi_{0}(t), \ldots, \phi_{B_{k}}(t)\right)^{\prime}, k=1,2, \ldots, p$. Then, the process $\boldsymbol{X}(t)$ can be represented as

$$
\boldsymbol{X}(t)=\boldsymbol{\Phi}(t) \mathbf{c}, t \in I, \mathrm{E}(\mathbf{c})=\mathbf{0}, \operatorname{Var}(\mathbf{c})=\boldsymbol{\Sigma}_{\mathbf{c}} .
$$

We are interested to find vector weight functions $\mathbf{u}(t)$ such that

$$
U=<\mathbf{u}(t), \boldsymbol{X}(t)>=\int_{I} \mathbf{u}^{\prime}(t) \boldsymbol{X}(t) d t
$$

has maximum variance for all $\mathbf{u}(t) \in L_{2}^{p}(I)$ such that $\langle\mathbf{u}(t), \mathbf{u}(t)\rangle=1$. It may be assumed that the vector weight function $\mathbf{u}(t)$ and the process $\boldsymbol{X}(t)$ are in the same space, i.e. the function $\mathbf{u}(t)$ can be written in the form:

$$
\mathbf{u}(t)=\boldsymbol{\Phi}(t) \mathbf{u},
$$

where fixed vector $\mathbf{u} \in \mathbb{R}^{K+p}, K=B_{1}+B_{2}+\ldots+B_{p}$. Then

$$
<\mathbf{u}(t), \boldsymbol{X}(t)>=<\boldsymbol{\Phi}(t) \mathbf{u}, \boldsymbol{\Phi}(t) \mathbf{c}>=\mathbf{u}^{\prime}<\boldsymbol{\Phi}(t), \boldsymbol{\Phi}(t)>\mathbf{c}=\mathbf{u}^{\prime} \mathbf{c}
$$

and

$$
\begin{aligned}
& \mathrm{E}(<\mathbf{u}(t), \boldsymbol{X}(t)>)=\mathbf{u}^{\prime} \mathrm{E}(\mathbf{c})=\mathbf{u}^{\prime} \mathbf{0}=0 \\
& \operatorname{Var}(<\mathbf{u}(t), \boldsymbol{X}(t)>)=\mathbf{u}^{\prime} \mathrm{E}\left(\mathbf{c} \mathbf{c}^{\prime}\right) \mathbf{u}=\mathbf{u}^{\prime} \boldsymbol{\Sigma}_{\mathbf{c}} \mathbf{u}
\end{aligned}
$$

Let

$$
\begin{aligned}
& \lambda_{1}=\sup _{\mathbf{u}(t) \in L_{2}^{p}(I)} \operatorname{Var}(<\mathbf{u}(t), \boldsymbol{X}(t)>)= \\
& \operatorname{Var}\left(<\mathbf{u}_{1}(t), \boldsymbol{X}(t)>\right)=\mathbf{u}_{1}^{\prime} \boldsymbol{\Sigma}_{\mathbf{c}} \mathbf{u}_{1},
\end{aligned}
$$


where $<\mathbf{u}_{1}(t), \mathbf{u}_{1}(t)>=\mathbf{u}_{1}^{\prime} \mathbf{u}_{1}=1$. As the first principal component will be called the inner product $U_{1}=<\mathbf{u}_{1}(t), \boldsymbol{X}(t)>=\mathbf{u}_{1}^{\prime} \mathbf{c}$, and the vector function $\mathbf{u}_{1}(t)$ will be called the first vector weight function. Subsequently we look for the second principal component $U_{2}=<\mathbf{u}_{2}(t), \boldsymbol{X}(t)>=\mathbf{u}_{2}^{\prime} \mathbf{c}$, maximizing $\operatorname{Var}(<\mathbf{u}(t), \boldsymbol{X}(t)>)=\mathbf{u}^{\prime} \boldsymbol{\Sigma}_{\mathbf{c}} \mathbf{u}$, such that $\left\langle\mathbf{u}_{2}(t), \mathbf{u}_{2}(t)\right\rangle=$ $\mathbf{u}_{2}^{\prime} \mathbf{u}_{2}=1$, and not correlated with the first functional principal component $U_{1}$, i.e. subject to the restriction $\left\langle\mathbf{u}_{1}(t), \mathbf{u}_{2}(t)\right\rangle=\mathbf{u}_{1}^{\prime} \mathbf{u}_{2}=0$.

In general, the $k$ th functional principal component $U_{k}=<$ $\mathbf{u}_{k}(t), \boldsymbol{X}(t)>=\mathbf{u}_{k}^{\prime} \mathbf{c}$ satisfies the conditions:

$$
\begin{aligned}
& \lambda_{k}=\sup _{\mathbf{u}(t) \in L_{2}^{p}(I)} \operatorname{Var}(<\mathbf{u}(t), \boldsymbol{X}(t)>)= \\
& \operatorname{Var}\left(<\mathbf{u}_{k}(t), \boldsymbol{X}(t)>\right)=\mathbf{u}_{k}^{\prime} \boldsymbol{\Sigma}_{\mathbf{c}} \mathbf{u}_{k}
\end{aligned}
$$

and

$$
<\mathbf{u}_{\kappa_{1}}(t), \mathbf{u}_{\kappa_{2}}(t)>=\delta_{\kappa_{1}, \kappa_{2}}, \quad \kappa_{1}, \kappa_{2}=1,2, \ldots, k .
$$

The expression $\left(\lambda_{k}, \mathbf{u}_{k}(t)\right)$ will be called the $k$ th principal system of the process $\boldsymbol{X}(t)$.

Now, let us consider the principal components of the random vector $\mathbf{c}$. The $k$ th principal component $U_{k}^{*}=<\mathbf{u}_{k}, \mathbf{c}>$ of this vector satisfies the conditions:

$$
\begin{aligned}
& \gamma_{k}=\sup _{\mathbf{u} \in \mathbb{R}^{\mathrm{K}+\mathrm{p}}} \operatorname{Var}(<\mathbf{u}, \mathbf{c}>)= \\
& \sup _{\mathbf{u} \in \mathbb{R}^{K+p}} \mathbf{u}^{\prime} \operatorname{Var}(\mathbf{c}) \mathbf{u}=\sup _{\mathbf{u} \in \mathbb{R}^{K+p}} \mathbf{u}^{\prime} \boldsymbol{\Sigma}_{\mathbf{c}} \mathbf{u}
\end{aligned}
$$

and

$$
\mathbf{u}_{\kappa_{1}}^{\prime}(t) \mathbf{u}_{\kappa_{2}}(t)=\delta_{\kappa_{1}, \kappa_{2}},
$$

where $\kappa_{1}, \kappa_{2}=1,2, \ldots, k, K=B_{1}+B_{2}+\ldots+B_{p}$. The expression $\left(\gamma_{k}, \mathbf{u}_{k}\right)$ will be called the $k$ th principal system of the vector $\mathbf{c}$.

In consequence, by determining the $k$ th principal system of $\mathbf{c}$, we obtain the $k$ th functional principal component of the process $\mathbf{X}(t)$, because $\lambda_{k}=\gamma_{k}$ and $\mathbf{u}_{k}(t)=\boldsymbol{\Phi}(t) \mathbf{u}_{k}$, where $k=1,2, \ldots, K+p, K=B_{1}+B_{2}+\ldots+B_{p}$.

Determining the $k$ th principal system of vector $\mathbf{c}$ is equivalent to solving for the eigenvalue and corresponding eigenvectors of the covariance matrix $\boldsymbol{\Sigma}_{\mathbf{c}}$ of that vector, standardized so that $\mathbf{u}_{\kappa_{1}}^{\prime}(t) \mathbf{u}_{\kappa_{2}}(t)=\delta_{\kappa_{1}, \kappa_{2}}$.

The covariance matrix $\boldsymbol{\Sigma}_{\mathbf{c}}$ can be estimated on the basis of $n$ independent realizations $\mathbf{x}_{1}(t), \mathbf{x}_{2}(t), \ldots, \mathbf{x}_{n}(t)$ of the random process $\boldsymbol{X}(t)$.

Typically data are recorded at discrete moments in time. The process of transformation of discrete data to functional data is performed for each 
variable $X_{1}, X_{2}, \ldots, X_{p}$ separately.

Let $x_{k j}$ denote an observed value of feature $X_{k}, k=1,2, \ldots, p$, at the $j$ th time point $t_{j}$, where $j=1,2, . ., J$. Then our data consist of $p J$ pairs $\left(t_{j}, x_{k j}\right)$. These discrete data can be smoothed by continuous functions $x_{k}(t)$, where $t \in I$ (Ramsay and Silverman, 2005). Let $I$ be a compact set such that $t_{j} \in I$, for $j=1,2, . ., J$. Let us assume that the function $x_{k}(t)$ has the following representation

$$
x_{k}(t)=\sum_{b=0}^{B_{k}} c_{k b} \phi_{b}(t), \quad t \in I, \quad k=1,2, \ldots, p,
$$

where $\left\{\phi_{b}\right\}$ are orthonormal basis functions, and $c_{k 0}, c_{k 1}, \ldots, c_{k B_{k}}$ are the coefficients.

Let $\mathbf{x}_{k}=\left(x_{k 1}, x_{k 2}, \ldots, x_{k J}\right)^{\prime}, \mathbf{c}_{k}=\left(c_{k 0}, c_{k 1}, \ldots, c_{k B_{k}}\right)^{\prime}$ and $\mathbf{\Phi}_{k}(t)$ be a matrix of dimension $J \times\left(B_{k}+1\right)$ containing the values $\phi_{b}(t), b=0,1, \ldots, B_{k}, j=1,2, . ., J, k=1,2, \ldots, p$. The coefficient $\mathbf{c}_{k}$ in (2) is estimated by the least squares method, that is, so as to minimize the function:

$$
S\left(\mathbf{c}_{k}\right)=\left(\mathbf{x}_{k}-\boldsymbol{\Phi}_{k}(t) \mathbf{c}_{k}\right)^{\prime}\left(\mathbf{x}_{k}-\mathbf{\Phi}_{k}(t) \mathbf{c}_{k}\right), \quad k=1,2, \ldots, p .
$$

Differentiating $S\left(\mathbf{c}_{k}\right)$ with respect to the vector $\mathbf{c}_{k}$, we obtain the least squares method estimator

$$
\hat{\mathbf{c}}_{k}=\left(\boldsymbol{\Phi}_{k}^{\prime}(t) \boldsymbol{\Phi}_{k}(t)\right)^{-1} \boldsymbol{\Phi}_{k}^{\prime}(t) \mathbf{x}_{k}, \quad k=1,2, \ldots, p .
$$

The degree of smoothness of the function $x_{k}(t)$ depends on the value $B_{k}$ (a small value of $B_{k}$ causes more smoothing of the curves). The optimum value for $B_{k}$ may be selected using the Bayesian information criterion (BIC); (see Schwarz (1978), Shmueli(2010)). Let us assume that there are $n$ independent pairs of values $\left(t_{j}, x_{k i j}\right), k=1,2, \ldots, p, i=1,2, \ldots, n$, $j=1,2, \ldots, J$. These discrete data are smoothed to continuous functions in the following form:

$$
x_{k i}(t)=\sum_{b=0}^{B_{k i}} \hat{c}_{k i b} \phi_{b}(t), \quad k=1,2, \ldots, p, \quad i=1,2, \ldots, n, \quad t \in I .
$$

Among all the $B_{k 1}, B_{k 2}, \ldots, B_{k n}$ one common value of $B_{k}$ is chosen, as the modal value of the numbers $B_{k 1}, B_{k 2}, \ldots, B_{k n}$, and we assume that each 
function $x_{k i}(t)$ has the form:

$$
x_{k i}(t)=\sum_{b=0}^{B_{k}} \hat{c}_{k i b} \phi_{b}(t), \quad k=1,2, \ldots, p, \quad i=1,2, \ldots, n, \quad t \in I .
$$

The data $\left\{x_{k 1}(t), \ldots, x_{k n}(t)\right\}$ are called functional data (see Ramsay and Silverman, 2005).

Finally, each of $n$ independent realizations $\mathbf{x}_{1}(t), \mathbf{x}_{2}(t), \ldots, \mathbf{x}_{n}(t)$ has the form $\mathbf{x}_{i}(t)=\boldsymbol{\Phi}(t) \hat{\mathbf{c}}_{i}$ where $\boldsymbol{\Phi}(t)$ is given by (1) and the vectors $\hat{\mathbf{c}}_{i}=$ $\left(\hat{c}_{10}, \ldots, \hat{c}_{1 B_{1}}, \ldots, \hat{c}_{p 0}, \ldots, \hat{c}_{p B_{p}}\right)$ are centered, $i=1,2, \ldots, n$.

Let $\hat{\mathbf{C}}=\left(\hat{\mathbf{c}}_{1}, \hat{\mathbf{c}}_{2}, \ldots, \hat{\mathbf{c}}_{n}\right)$. Then

$$
\hat{\mathbf{\Sigma}}_{\mathbf{c}}=\frac{1}{n} \hat{\mathbf{C}} \hat{\mathbf{C}}^{\prime}
$$

Let $\hat{\gamma}_{1} \geqslant \hat{\gamma}_{2} \geqslant \ldots \geqslant \hat{\gamma}_{s}$ be non-zero eigenvalues of the matrix $\hat{\boldsymbol{\Sigma}}_{\mathbf{c}}$, and $\hat{\mathbf{u}}_{1}, \hat{\mathbf{u}}_{2}, \ldots, \hat{\mathbf{u}}_{s}$ the corresponding eigenvectors, where $s=\operatorname{rank}\left(\hat{\boldsymbol{\Sigma}}_{\mathbf{c}}\right)$.

Moreover, the $k$ th principal system of the random process $\boldsymbol{X}(t)$ determined from the sample has the following form:

$$
\left(\hat{\lambda}_{k}=\hat{\gamma}_{k}, \hat{\mathbf{u}}_{k}(t)=\boldsymbol{\Phi}(t) \hat{\mathbf{u}}_{k}\right), \quad k=1,2, \ldots, s .
$$

The coordinates of the projection of the $i$ th realization $\mathbf{x}_{i}(t)$ of process $\boldsymbol{X}(t)$ on the $k$ th functional principal component are equal to:

$$
\begin{aligned}
& \hat{U}_{i k}=<\hat{\mathbf{u}}_{k}(t), \mathbf{x}_{i}(t)>=<\boldsymbol{\Phi}(t) \hat{\mathbf{u}}_{k}, \boldsymbol{\Phi}(t) \hat{\mathbf{c}}_{i}^{\prime}>= \\
& \hat{\mathbf{u}}_{k}^{\prime}<\boldsymbol{\Phi}(t), \boldsymbol{\Phi}(t)>\hat{\mathbf{c}}_{i}^{\prime}=\hat{\mathbf{u}}_{k}^{\prime} \hat{\mathbf{c}}_{i}^{\prime},
\end{aligned}
$$

for $i=1,2, \ldots, n, k=1,2, \ldots, s$. Finally the coordinates of the projection of the $i$ th realization $\mathbf{x}_{i}(t)$ of process $\boldsymbol{X}(t)$ on the plane of the first two functional principal components from the sample are equal to $\left(\hat{\mathbf{u}}_{1}^{\prime} \hat{\mathbf{c}}_{i}^{\prime}, \hat{\mathbf{u}}_{2}^{\prime} \hat{\mathbf{c}}_{i}^{\prime}\right)$, $i=1,2, \ldots, n$.

\section{Calculation results}

The analysis relates to 12 statistical objects $(n=12)$, i.e. three treatments each in four replications. Each object was characterized by a group of 14 properties $(p=14)$. The value of each property was measured at 20 time points $(J=20)$. In the first step, the original data were transformed to functional data by the method described in section 3 . The calculations were performed using the Fourier basis system, which is a typical selection, although others such as splines, polynomials and wavelets can also be used. 
We used the R package fda (Ramsay et al. 2014) to create a Fourier system in order to convert raw data into a functional object. The optimum degrees of smoothness were selected using the BIC criterion; for details see Table 1. In the Figure 1, selected graphs showing the results of transformation are given.

Table 1. Chosen levels of smoothing.

\begin{tabular}{lcr}
\hline No. & Statistical variables & $B_{k}$ \\
\hline 1. & $\mathrm{pH}$ & 5 \\
2. & $\mathrm{BS}$ & 11 \\
3. & $\mathrm{Hh}$ & 3 \\
4. & $\mathrm{Corg}$ & 11 \\
5. & $\mathrm{Ntot}$ & 7 \\
6. & $\mathrm{Nmin}$ & 3 \\
7. & $\mathrm{P}$ & 5 \\
8. & $\mathrm{~K}$ & 5 \\
9. & $\mathrm{Mg}$ & 9 \\
10. & $\mathrm{Fe}$ & 7 \\
11. & $\mathrm{Mn}$ & 5 \\
12. & $\mathrm{Zn}$ & 11 \\
13. & $\mathrm{Cu}$ & 3 \\
14. & $\mathrm{Ni}$ & 3 \\
\hline
\end{tabular}

We also implemented other procedures. In the second step, we construct the functional principal components. The system of the first two functional principal components retains $67.08 \%$ of the total variation.

The contribution of the original variables to the construction of the individual principal components is not the same. At a given time point $t$, a greater contribution to the structure of a given functional principal component comes from the variable $X$ corresponding to that component. The total contribution of a particular original variable $X_{i}$ to the structure of a given functional principal component is equal to the area under the module weighting function corresponding to that variable. These contributions, for 14 variables, and the first and second functional principal components, are given in Table 2 .

In the final step we form homogeneous groups of objects applying cluster analysis. We want to divide 12 objects into groups in such a way that 

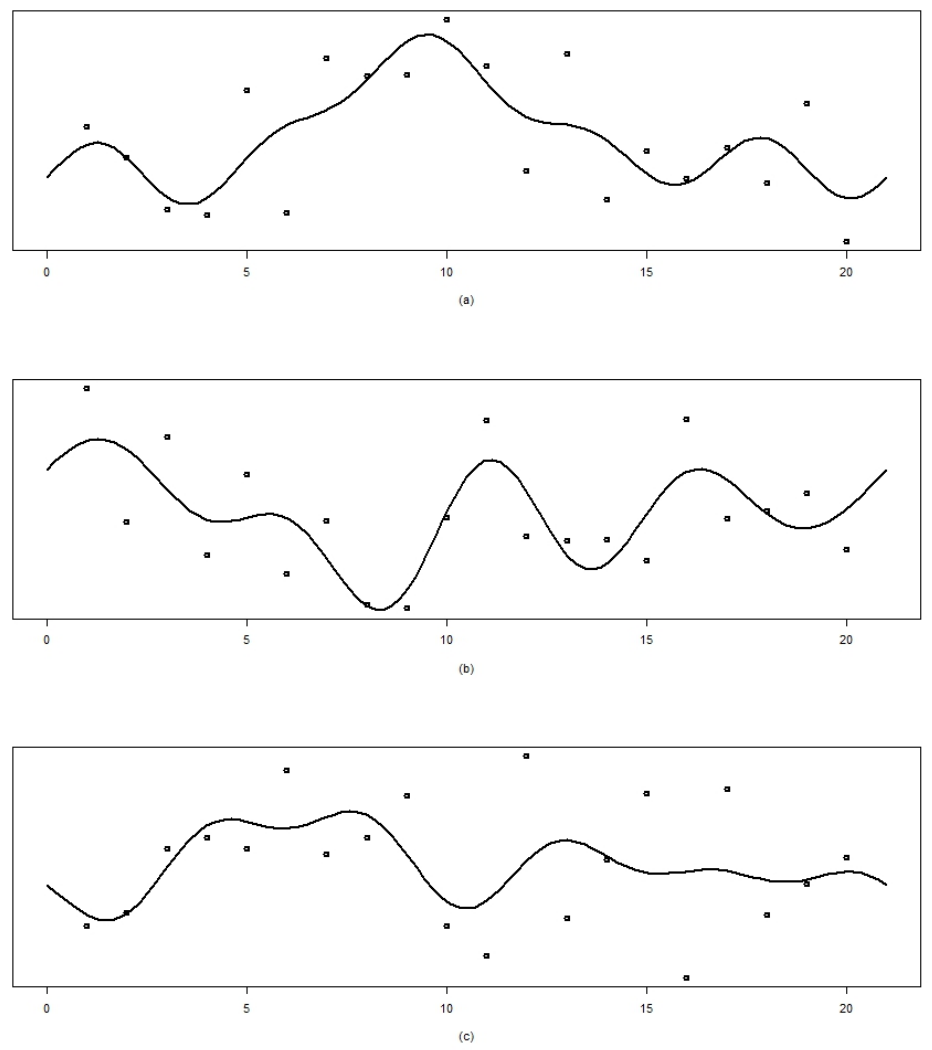

Figure 1. Original and transformed data (Corg). (a) control soil;

(b) straw with stem filled by pith; (c) straw with hollow stem.

objects belonging to the same group do not significantly differ with respect to the chosen distance. In the first step, we construct the minimum spanning tree (Florek et al. 1951). Two popular algorithms for constructing the minimum spanning tree are the Kruskal algorithm (Kruskal 1956) and the Prim algorithm (Prim 1957). Kruskal's algorithm is a greedy algorithm that adds edges in order of their lengths, so long as they do not result in a cycle. Prim's algorithm starts with a tree consisting of a single vertex, and keeps adding the smallest edge of the tree. In our computations we used the Kruskal algorithm. The minimum spanning tree can be used for clustering. The idea is to break (remove edges from) the minimum spanning tree at edges that are greater than the common critical value $d_{1}$. We compute the 
mean $\bar{x}$ and the standard deviation $s$ of all edge lengths in the minimum spanning tree, and eliminate edges which are longer than $d_{1}=\bar{x}+s$. In our case the mean $\bar{x}$ and the standard deviation $s$ are equal to $\bar{x}=0.96$ and $s=1.31$. The value of the common critical value $d_{1}$ is 2.27 . The relative positions of the 12 objects in the system of the first two functional principal components, with the minimum spanning tree, are shown in Figure 2. The points which remain connected in the minimum spanning tree form clusters. From Figure 2, we see that the 12 objects form three homogeneous groups: control soil, soil incubated with straw with stem filled by pith and soil incubated with straw with hollow stem. Note moreover that the distance between objects 1 and 5 is greater than the critical value $d_{2}=\bar{x}+2 s$. Thus, the difference between the group of objects with normal soil and the group with full stemmed straw is highly significant.

Table 2. The ratio of the area under the module of a given weighting function to the sum of areas under the module for all weighting functions (in \%).

\begin{tabular}{lrrr}
\hline No. & Statistical variables & Comp. 1 & Comp. 2 \\
\hline 1. & $\mathrm{pH}$ & 5.77 & 7.55 \\
2. & $\mathrm{BS}$ & 8.27 & 11.08 \\
3. & $\mathrm{Hh}$ & 5.20 & 4.89 \\
4. & $\mathrm{Corg}$ & 19.83 & 4.43 \\
5. & $\mathrm{Ntot}$ & 5.59 & 9.34 \\
6. & $\mathrm{Nmin}$ & 10.83 & 8.36 \\
7. & $\mathrm{P}$ & 5.67 & 3.89 \\
8. & $\mathrm{~K}$ & 8.07 & 5.18 \\
9. & $\mathrm{Mg}$ & 7.14 & 13.47 \\
10. & $\mathrm{Fe}$ & 4.72 & 7.75 \\
11. & $\mathrm{Mn}$ & 6.08 & 9.08 \\
12. & $\mathrm{Zn}$ & 6.80 & 9.14 \\
13. & $\mathrm{Cu}$ & 2.15 & 2.16 \\
14. & $\mathrm{Ni}$ & 3.89 & 3.67 \\
\hline
\end{tabular}

\section{Discussion}

The first functional principal component describes $48.53 \%$ and the second functional principal component $18.55 \%$ of the variability of soil properties. Each principal component is the weighted sum of 14 properties and we 


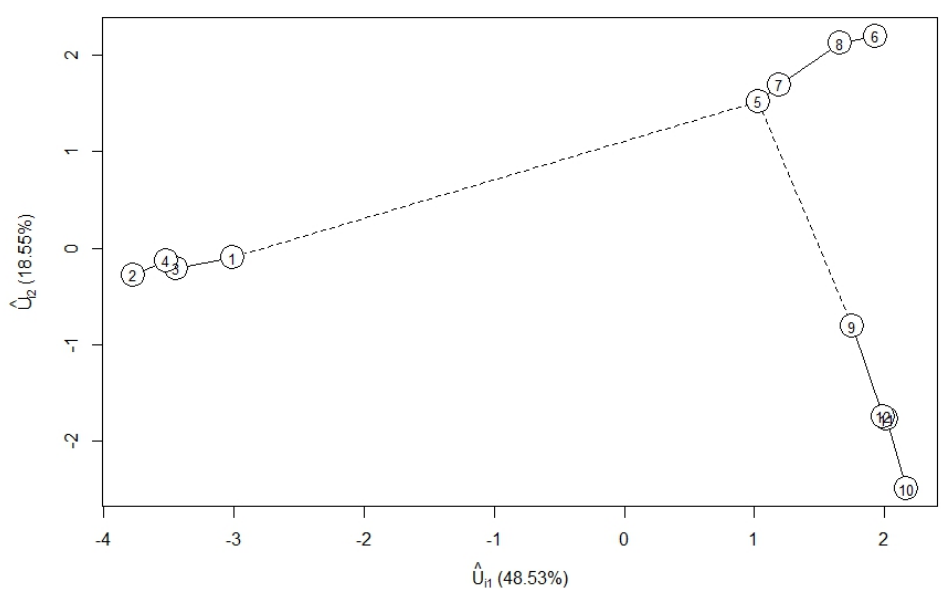

Figure 2. The relative positions of the 12 objects in the system of the first two functional principal components, with the minimum spanning tree.

wish to indicate which of them is the most significant. For each functional principal component, we choose three properties that have a large influence on the variation between soils. Corg has a $19.83 \%$ impact on the first functional principal component, Nmin $10.83 \%$ and BS $8.27 \%$. In the case of the second functional principal component, $\mathrm{Mg}$ has a $13.47 \%$ impact, BS $11.08 \%$ and Ntot $9.34 \%$.

The location of the 12 objects in the system of the first two functional principal components indicated that addition of the straws, independently of the degree of filling of the stems by pith, did not cause significant variation in the soil properties. However, differences between the control soil and soil incubated with the straws were observed. This differentiation was expressed by the changes in organic carbon content, mineral nitrogen content and sum of bases recorded during the incubation process (Figure 2). At the same time, the type of straw and the related varying quantities of hard decomposed substances such as cellulose, hemicellulose and lignin did not significantly influence the rate of mineralization of organic matter in the soil (Figure 2). A possible explanation may be that the share of these substances in stems filled by pith was not so considerable and was comparable to the concentrations in hollow stems. Accordingly, the rate of 


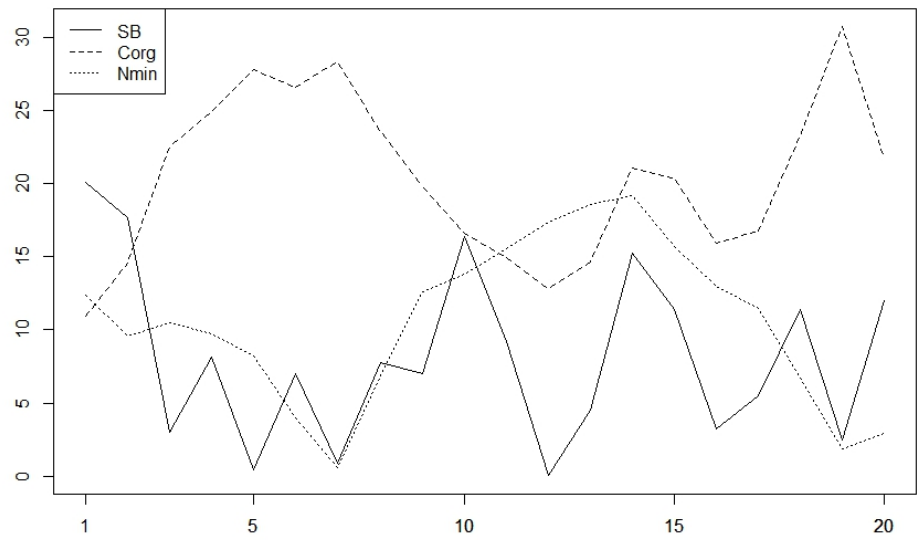

Figure 3. The ratio of the area under the module of a given weighting function to the sum of areas under the module for all weighting functions, for the first functional principal component for BS, Corg, Nmin (in \%).

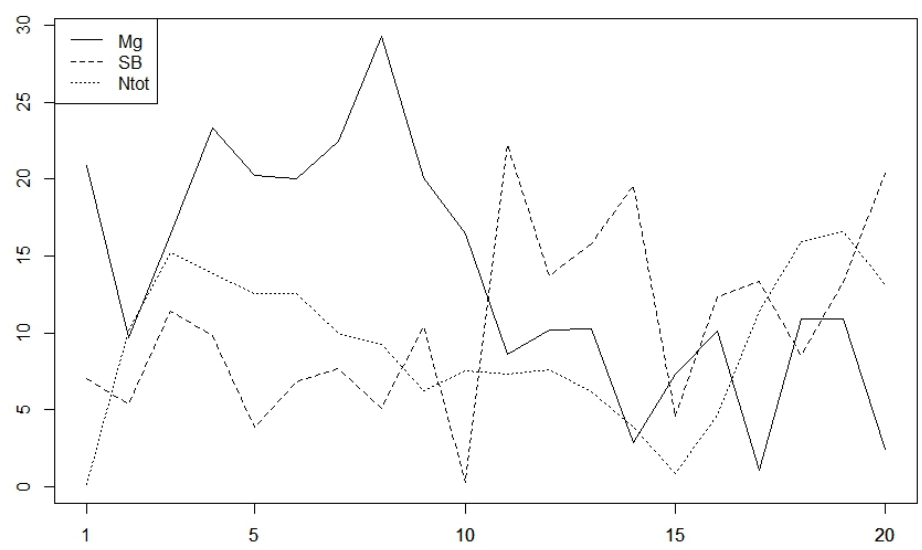

Figure 4. The ratio of the area under the module of a given weighting function to the sum of areas under the module for all weighting functions, for the second functional principal component for $\mathrm{Mg}$, BS, Norg (in \%). 
decomposition of these straws showed the same pattern. Regardless of the degree of stem solidness, the maximum amounts of Corg, Nmin and sum of bases were assessed at the end of the incubation experiment (14, 15 terms). These confirm the slow mineralization of the straws. Ogunniyi et al. (2014) indicated that the relatively slow decomposition of straw is caused by its high lignin, cellulose and hemicellulose contents. Chen et al. (2014) added that effective straw mineralization depends on the activity of fungi and the intensity of their extracellular depolymerizing enzymes. Organic matter introduced into the soil in the form of straw had a considerable impact not only on the contents of organic carbon, but also on the sum of bases. As we can see from Figures 3 and 4, the values of SB significantly differentiated the control soil and soil incubated with the two types of straw, as well as the soil incubated with straw with stem filled by pith and the soil incubated with straw with hollow stem. Although the decomposition of organic matter causes decreasing $\mathrm{pH}$ with accompanying increasing hydrolytic acidity (Hh), the data in Table 1 show that $\mathrm{pH}$ and $\mathrm{Hh}$ explained the variability of soil properties to degrees of only $5.77 \%$ and $5.20 \%$ respectively. This can be explained by the presence of organic matter as a strong buffering factor. Additionally, the maintenance of proper soil reaction depends on buffer capacity, which is related to high values of SB and amounts of magnesium. Rezig et al. (2014) proved that the application of nitrogen and phosphorous is an important management practice to enhance the decomposition of high $\mathrm{C}: \mathrm{N}$ crop residues (for example straw) in the soil. Taking into consideration the above information, the results obtained from the incubation experiment partly confirmed such a tendency.

The influence of SB and Nmin (Figure 3) as well as SB and $\mathrm{Mg}$ (Figure 4) on differences between soil treatments (T0 versus $\mathrm{T} 1$ and $\mathrm{T} 2$ ) can be explained by the importance of applying straw in controlling the proper soil reaction and rate of mineralization of supplied organic matter. Moreover, the degree to which the stems of winter wheat were filled by pith was not significant.

\section{Conclusions}

1. Although, by use of principal functional component analysis, $67.08 \%$ of the total variation of soil properties is retained, this analysis is a very suitable tool for explaining the relations and variation between the control soil and the soil incubated with the straws. 
2. The content of organic carbon, mineral nitrogen and sum of bases played the major role in describing the variability of soil properties and the differences between experimental treatments.

3. The study has confirmed that straw, independently of the degree of filling of winter wheat stems by pith, may act as a slow decomposing crop residue.

\section{Acknowledgements}

The authors thank the anonymous reviewer for his comments and suggestions that resulted in improvement in the quality of presentation of the manuscript.

\section{REFERENCES}

Chen L., Zhang J.B., Zhao B.Z., Xin X.L., Zhou G.X., Tan J.F., Zhao J.H. (2014): Carbon mineralization and microbial attributes in straw-amended soils as affected by moisture levels. Pedosphere 24(2): 167-177.

Florek K., Łukaszewicz J., Perkal J., Steinhaus H., Zubrzycki S. (1951): Sur la liaison et la division des points d'un ensemble fini. Colloquium Mathematicum 2: $282-285$.

Górecki T., Krzyśko M. (2012): Functional Principal Components Analysis, Data analysis methods and its applications. C.H. Beck: 71-87.

IUSS Working Group WRB (2007): World Reference Base for Soil Resources 2006, first update 2007. World Soil Resources Reports, 103. FAO, Rome.

Jacques J., Preda C. (2014): Model-based clustering for multivariate functional data. Computational Statistics \& Data Analysis 71: 92-106.

Jakubus M. (2013): Wybrane zagadnienia z gleboznawstwa i chemii rolnej. Wydanie II rozszerzone i uaktualnione, Poznan, Uniwersytet Przyrodniczy w Poznaniu. [In Polish].

Kruskal J.B. (1956): On the shortest spanning subtree of a graph and the travelling salesman problem. Proceedings of American Mathematical Society 7: 48-50.

Ogunniyi J.E., Guo C. Tian X.H., Li H.Y., Zhou Y.X. (2014): The effects of three mineral nitrogen sources and zinc on maize and wheat straw decomposition and soil organic carbon. Journal of Integrative Agriculture 13(12): 2768-2777.

Prim R.C. (1957): Shortest connection networks and some generalizations. Bell System Technical Journal 36: 1389-1401.

Ramsay J.O., Silverman B.W. (2005): Functional Data Analysis. Second Edition, Springer, New York. 
Ramsay J.O., Wickham H., Graves S., Hooker G. (2014): fda: Functional Data Analysis. R package version 2.4.4. https: //CRAN. R-project.org/package= fda.

Rezig F.A.M., Elhadi E.A., Abdalla M.R. (2014): Decomposition and nutrient release pattern of wheat (Triticum aestivum) residues under different treatments in desert field conditions of Sudan. Int. J. Recycl. Org. Waste Agricult. 3, 69: DOI 10.1007/s40093-014-0069-8.

Shmueli G. (2010): To explain or to predict? Statistical Science 25(3): 289-310.

Schwarz G. (1978): Estimating the dimension of a model. Annals of Statistics 6(2): 461-464.

Thematic strategy for soil protection. Commission staff working document. Communication from the commission to the council, the European parliament, the European economic and social committee and the committee of the regions. COM (2006)231. 